\title{
Nurses' Knowledge and Attitudes about Adult Post-operative Pain Assessment and Management: A Cross-Sectional Study in Qatar
}

Ms Haya Samara, Master of Public Health student, Qatar University

Supervisor: Dr. Lily O'Hara, Associate Professor of Public Health, Qatar University

\section{Introduction}

Worldwide, pain is considered a major health problem. ${ }^{1}$ Clinicians, patients, and researchers alike describe "pain" as a complex and challenging phenomenon. The International Association for the Study of Pain defines pain as "an unpleasant sensory and emotional experience associated with actual or potential tissue damage or described in terms of such damage." 2,3

Pain can also be defined subjectively as the person's reported experience, where each person feels and reports it differently. ${ }^{2}$ People may experience acute or chronic pain or a combination of both. ${ }^{4}$ It is vital that hospital based nurses are knowledgeable and have appropriate attitudes towards the assessment and management of pain.

Various international studies have demonstrated that nurses' knowledge and attitudes (K\&A) about pain assessment and management are lacking. ${ }^{5}$ However, this issue has not been studied in Qatar.

\section{Aim and Objectives}

The aim of this study was to assess the knowledge and attitudes of nurses in Qatar about adult post-operative patients' pain assessment and management and the factors that may be associated with such knowledge and attitudes.

\section{Objective 1}

To determine the level of nurses' knowledge and attitudes about adult post-operative patients' pain assessment and management.

\section{Objective 2}

To determine the association between nurses' knowledge and attitudes about adult post-operative patients' pain assessment and management and sociodemographic factors.

\section{Objective 3}

To determine the association between nurses' knowledge and attitudes about adult post-operative patients' pain assessment and management and previous attendance at pain education programs.

\section{Methods}

The study involved a cross-sectional online survey of all nurses providing bedside care in adult post-operative areas in Hamad Medical Corporation (HMC). Participants completed the Knowledge and Attitudes Survey Regarding Pain tool, ${ }^{3}$ hours of pain education, and a sociodemographic questionnaire. The outcome for analysis was the total K\&A score. The study was approved by the QU-IRB, approval number 1451EA/21 and the HMC MRC approval number MRC-01-20-796-DSA.

\section{Statistical Analysis}

Descriptive analysis: frequency for categorical variables; mean, range, and standard deviation for continuous variables

Inferential analysis: t-test and ANOVA to test for correlation between $K \& A$ score and independent variables

\section{Results}

Participants included 151 post-operative nurses (females $=70 \%$ ). Most of the respondents were married $(71 \%)$ and non-Qatari $(97 \%)$. The mean age was 37 years (SD 7.8) and the mean years of experience as nurse was 13.5 years (SD 7.1). Most respondents had a bachelor's degree (77\%) and had received 1-2 hours (37\%) or 3-5 hours $(27 \%)$ of pain education in the last two years. Over half the respondents worked at Hazem Mubiereek General Hospital (27\%) or Hamad General Hospital (26\%), and most respondents were staff nurses $(76 \%)$.

The mean knowledge and attitude (K\&A) score was 19.6 (SD 4.5) out of $41(48 \%)$ with a range of 8 to $32(19.5 \%$ to $78.0 \%)$.

$K \& A$ scores increased with higher levels of education, but differences were not statistically significant $(F=2.08, P=0.13)$

$K \& A$ scores were not associated with marital status $(F=0.35, P=0.78$

K\&A scores were not associated with participants' facility ( $F=0.82$ $K \& A$ scores were not associated with participants
$P=0.55)$ or job designation $(F=0.88, P$ value $=0.51)$

There was no correlation between $K \& A$ scores and age $(r=-0.01$ $P=0.88)$ or years of experience $(r=-0.03, P=0.79)$ 1

Hours spent in pain education were not associated with K\&A scores $(F=1.01, P=0.40)$

$K \& A$ scores were not associated with nationalities $(t=1.92, P=0.06,95 \% \mathrm{Cl}$ : $-0.14,10.22)$ or with gender $(t=-1.8431, P=0.06,95 \% \mathrm{Cl}:-3.35,0.12)$

\section{Conclusion}

The study findings indicated a significant deficit in nurses' knowledge and attitudes about adult post-operative pain. Knowledge and attitudes were not associated with any sociodemographic factors or with hours of previous pain education. This suggests that the deficit is widespread across nurses in general. The findings are consistent with studies elsewhere that revealed that nurses have poor knowledge and attitudes regarding pain.

Further research is required to understand the qualitative experiences of nurses in pain assessment and management, and to inform professional development strategies to enhance nurses' knowledge and attitudes. Such programs must be tailored to the nurses' characteristics and settings.

\section{References}

1. Kahsay, H. (2017). Assessment and treatment of pain in pediatric patients. Current Pediatric Research.

2. Dames, L. J. P., Alves, V. H., Rodrigues, D. P., de Souza, R. R. B., Medeiros, F. d. V. A., \& Paiva, E. D. (2016). Nurses' practical knowledge on the clinical management of neonatal pain: a descriptive study. Online Brazilian Journal of Nursing, 15(3), 393-403.

3. B. Ferrell and M. McCaffery, "Knowledge and attitude survey regarding pain," 2014, http://prccohorg.

4. Eaton, L. H., Meins, A. R., Mitchell, P. H., Voss, J., \& Doorenbos, A. Z. (2015). Evidencebased practice beliefs and behaviors of nurses providing cancer pain management: a mixed-methods approach. Oncology nursing forum, 42(2), 165-173.

doi:10.1188/15.onf.165-173

5. Treede, R.-D. (2018). The International Association for the Study of Pain definition of pain: as valid in 2018 as in 1979, but in need of regularly updated footnotes. Pain reports, 3(2) 